\title{
Estimation for two exponential life time models under joint multiply type-II censoring
}

Neha K. Gadhvi $i^{*}$

\section{*Correspondence:}

nehakgadhvi@gmail.com

Technology, Operations

and Decision Sciences,

Ahmedabad University,

Ahmedabad, Gujarat 380009,

India

\begin{abstract}
Various types of censoring schemes basically type-l and type-II censoring schemes and their modified versions are used in life testing experiments. Most of the tests used in life testing experiments are based on a single sample. A joint censoring scheme is quite useful in conducting comparative life tests of products from different units within the same facility. In this article, we consider two exponential life time models under joint multiply type-II censoring scheme, which is a generalization of usual type-II censoring scheme, implemented on the two samples. We have considered maximum likelihood estimation and Bayesian estimation for estimating the reliability of the product under such a censoring scheme. The results are compared with the results obtained under usual type-II censoring scheme. In Bayes estimation the effect of prior parameters on mean life time and reliability of the product is discussed. We have used the local influence approach for identifying observations that strike a disproportionate effect in the maximum likelihood estimate of the reliability in the model. The life time data set of air-conditioning systems of two Boeing 720 jet airplanes "7914" and "7913" are used to apply the theory developed in the paper.
\end{abstract}

Keywords: Maximum likelihood estimation, Asymptotic variance, Influence measure, Inverse gamma prior, Bayes estimation

Mathematics Subject Classification: 62F15, 62F30, 62N02, 62N05

\section{Introduction}

Different censoring schemes are available in the literature to optimize time, cost and efficiency of the life testing experiment. Among different censoring schemes, type-I and type-II are the two most popular censoring schemes. In a type-I censoring scheme, the experiment is terminated at a prefixed time point. In type-I censoring, number of failures is purely random. So, it may happened that the observed number of failures during the pre-determined fixed time may be very small or sometimes zero which will lead to a biased or inefficient statistical analysis of associated model parameters. To get a certain number of failures, type-II censoring scheme is used. But in type-II censoring scheme, the test termination time is random and it may takes more than the expectation of the experimenter, which increases the cost of the experiment. The work done under such types of censoring schemes is mostly on the basis of single censored sample. 
However, in practical market, there are situations in which the experimenter wishes to compare the product produced by different producers under the same facility. From a practical point of view, it is necessary to identify one item in presence of the other relative items. This problem requires sampling of the different production lines, and then the joint censoring scheme is appeared. To this end, the reliability comparative life test is apparently desirable. In order to guarantee the same experimental condition that items are exposed to, a joint censoring model is used to describe the test model.

Recently, two sample joint censoring schemes becoming popular for a life testing experiment mainly to optimize time and cost. Two independent samples of sizes $m$ and $n$ are selected from these lines and put simultaneously on a life testing experiment. Then, the experimenter follows a joint complete sample scheme or a joint censoring scheme and terminates the life testing experiment when a pre-determined number of failures (say, $r$ ) occur, which is known as joint type-II censoring.

To determine reliability of a product, life time distribution plays an important role. Selection of a proper life time model for the product is an art. In literature various types of life time models are available like: exponential, Weibull, Rayleigh, gamma, logistic, Pareto, Kumaraswamy etc. The life time model can be selected on the basis of the failure rate of the product. Exponential model is used for the product having constant failure rate. Weibull life time model is a very general life time model which can be used when the product has decreasing, constant or an increasing failure rate.

Exponential life time model is such a life time model which possesses many interesting properties and very much mathematically tractable. Life times of many electrical and electronic items follow exponential distribution. In this paper we have utilized exponential life time model. A lot of work has been done based on exponential life time model under various types of censoring schemes.

In the literature, joint type-II censoring scheme, joint progressive type-II censoring scheme and statistical analysis based upon such schemes have been discussed earlier. Balakrishnan and Rasouli [1] developed likelihood inference for the parameters of two exponential populations under joint type-II censoring. They developed inferential methods based on maximum likelihood estimates and compared their performance with those based on some other approaches such as Bootstrap. Shafay et al. [2] derived the Bayesian inference for the unknown parameters of two exponential populations under joint type II censoring. They developed the estimates with the use of squared-error, linear-exponential and general entropy loss functions.

Balakrishnan and Feng [3] generalized Balakrishnan and Rasouli [1] and Shafay et al. [2] works by considering a jointly type-II censored sample arising from $h$ independent exponential populations. Ashour and Abo-Kasem [4, 5] derived Bayesian and nonBayesian estimators for two generalized exponential populations under joint type-II censored scheme.

Parsi and Bairamov [6] extended the scheme of two joint type-II censoring to a joint progressive type-II censoring scheme and considered expected values of the number of failures for two populations under joint type-II progressive censoring. Rasouli and Balakrishnan [7] provided the exact likelihood inference for two exponential populations under this censoring scheme. They have also derived the exact lower confidence 
bounds, exact confidence intervals, and simultaneous confidence regions for the parameters of the model based on classical and Bayesian setup.

Balakrishnan and $\mathrm{Su}$ et al. [8] extended the joint progressive censoring model to general $\mathrm{K}$ population model and studied exact likelihood inference of the unknown parameters for exponential distributions. Nadi and Gildeh [9] used progressive firstfailure censored sample for estimating the lifetime performance index of products for two-parameter exponential distribution.

Mondal and Kundu [10] recently introduced a balanced joint progressive type-II censoring scheme and provided the exact likelihood inference for the two exponential populations. It is observed that it has certain advantages over the joint progressive censoring scheme originally introduced by Rasouli and Balakrishnan [7].

In this paper we suggest a joint multiply type-II censoring scheme for the product having two exponential life time distributions. The rest of the paper is organized as follows: In "Methods" section, we first introduce the multiply type-II censoring and then it is utilized for development of joint multiply type-II censoring model and its likelihood function. Maximum likelihood estimates and their asymptotic standard errors of the mean life time and reliability of the two similar line products are derived. Based on the asymptotic normal distributions of maximum likelihood estimators, asymptotic confidence intervals for mean life times of the products are derived. Then after an influence measure is considered, by which the influence of individual observations on maximum likelihood estimates can be determined. Bayes estimation of mean life times and reliabilities of the products are also discussed. In "Results and discussion" section, the real data set regarding air-conditioning system of a fleet of 13 Boeing 720 jet airplanes considered by Proschan [11] is used to exemplify the results obtained in the earlier sections. Finally overall conclusion is given in "Conclusion" section.

\section{Methods}

\section{Multiply type-II censoring}

A generalization of type-II censoring scheme is known as multiply type-II censoring scheme. The following two types of multiply type-II censoring schemes are available:

- Under this scheme, from $\mathrm{n}$ items on the test we observe only the $r_{1}$-th, $r_{2}$ th, $\cdots, r_{k}$-th failure times $X_{r_{1}}, X_{r_{2}}, \ldots, X_{r_{k}}$, where $1 \leq r_{1}<r_{2}<\cdots<r_{k} \leq n$, and the rest of the data are not available.

- In life testing experiments the test is terminated either at predetermined time observed (type-I censoring) or at a predetermined number of failures observed (type-II censoring). Such censoring schemes may be from left or right. Sometimes left and right censoring appears together, this is known as doubly censoring. Furthermore, if mid censoring arises amongst the doubly censoring in the type II censoring scheme, the scheme is also known as multiply type-II censoring.

Applications of multiply type-II censoring scheme are available in literature. Some of the references related to the multiply type-II censoring scheme described in (a) are 
Balakrishnan [12], Balakrishnan et al. [13], Shah and Patel [14], Kim and Han [15], and in (b) are Upadhyay et al. [16], Kong [17], Shah and Patel [18], Kang et al. [19], Patel and Patel [20] and Shafay and Sultan [21].

\section{Joint multiply type-II censoring}

Let there are two lines of similar products and our aim is to study the relative merits of these two products. A sample of size $m$ is drawn from one product line, called type-A and another sample of size $n$ is drawn from the other product line, called type-B.

Suppose that, $Y_{1}, Y_{2}, \ldots, Y_{m}$ the lifetimes of $m$ specimens of product type- $A$, are independent identically distributed random variables from distribution function $F(y)$ and density function $f(y)$, and $W_{1}, W_{2}, \ldots, W_{n}$, the lifetimes of $n$ specimens of product type$\mathrm{B}$, are also independent identically distributed random variables from distribution function $G(w)$ and density function $g(w)$. Further, suppose $X_{1}<X_{2}<\cdots<X_{N}$ denote the order statistics of the $N=m+n$ random variables $\left\{Y_{1}, Y_{2}, \ldots, Y_{m} ; W_{1}, W_{2}, \ldots, W_{n}\right\}$. Here we assume that the probability distributions of $Y$ and $W$ are continuous, a unique ordering is always possible, since theoretical ties do not exist. Even though, if two observations from $Y$ and $W$ are equal, we can break the tie using random arrangement of these observations.

Suppose that only $k$ ordered failure times $X_{r_{1}}, X_{r_{2}}, \ldots, X_{r_{k}}$ are observed out of $X_{1}, X_{2}, \ldots, X_{N}$ ordered failure times. Here experimenter pre-fixes the values of $r_{1}, r_{2}, \ldots, r_{k}$ before conducting the life testing experiment. In this scheme, initial $r_{1}-1$ failures, some intermediate failures and last $N-r_{k}$ failures are not observed, since our aim is to save time and cost of the experiment. The joint multiply type-II censoring scheme can be visualized graphically as follows.

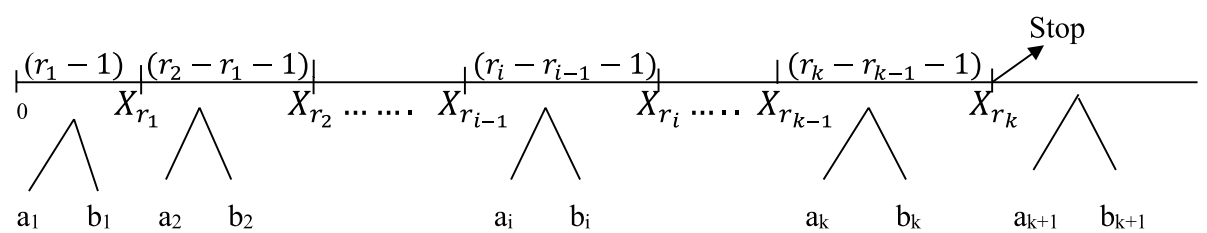

where $a_{\mathrm{i}}=$ number of failures of type-A with $X_{r_{i-1}}<Y<X_{r_{i}}, i=1,2, \ldots, k ; r_{0}=0, X_{0}=0$; $b_{i}=$ number of failures of type-B with $X_{r_{i-1}}<W<X_{r_{i}}, i=1,2, \ldots, k ; a_{k+1}=$ number of failures of type-A with $X_{r_{k}}<Y=m-m_{k}-\sum_{i=1}^{k} a_{i}$; $b_{k+1}=$ number of failures of typeB with $X_{r_{k}}<W=n-n_{k}-\sum_{i=1}^{k} b_{i}=R_{k+1}-a_{k+1}$; Let $m_{k}=$ number of failures of type-A out of the $\mathrm{k}$ observed failures $=\sum_{i=1}^{k} Z_{i} ; n_{k}=$ number of failures of type-B out of the $\mathrm{k}$ observed failures $=\sum_{i=1}^{k}\left(1-Z_{i}\right)=k-m_{k}$; Note that $m_{k}$ and $n_{k}$ both simultaneously cannot be zero. If any one of them is zero the problem reduces to estimation based on single sample only.

$$
\text { Denote } R_{1}=r_{1}-1, R_{i}=r_{i}-r_{i-1}-1, i=1,2, \ldots, k \text { and } R_{k+1}=a_{k+1}+b_{k+1}
$$

Then, under the joint multiply type-II censoring scheme, the observable data consist of $(Z, X)$, where

$$
\begin{gathered}
X=\left\{X_{r_{1}}, X_{r_{2}}, \ldots, X_{r_{k}}\right\}, \text { with } 1<r_{1}<r_{2}<\cdots<r_{k}<N \text {, and } \\
Z=\left\{Z_{1}, Z_{2}, \ldots, Z_{k}\right\} \text { with } Z_{i}=1 \text { or } 0 \text { according to } Z_{i} \text { is from } Y \text { or } W \text { failure. }
\end{gathered}
$$


Then the likelihood function of $(Z, X)$ will be given by

$$
\begin{aligned}
L= & C\left\{F\left(x_{r_{1}}\right)\right\}^{a_{1}}\left\{G\left(x_{r_{1}}\right)\right\}^{b_{1}} \prod_{i=1}^{k}\left\{f\left(x_{r_{i}}\right)\right\}^{z_{i}} \prod_{i=1}^{k}\left\{g\left(x_{r_{i}}\right)\right\}^{1-z_{i}} \prod_{i=2}^{k}\left[F\left(x_{r_{i}}\right)-F\left(x_{r_{i-1}}\right)\right]^{a_{i}} \\
& \times \prod_{i=2}^{k}\left[G\left(x_{r_{i}}\right)-\left(x_{r_{i-1}}\right)\right]^{b_{i}}\left\{\bar{F}\left(X_{r_{k}}\right)\right\}^{a_{k+1}}\left\{\bar{G}\left(X_{r_{k}}\right)\right\}^{b_{k+1}}
\end{aligned}
$$

where $C=\frac{m ! n !}{\prod_{i=1}^{k+1} a_{i} ! \prod_{i=1}^{k+1} b_{i} !}$.

Consider exponential life time models for life time $Y$ and $W$ as

$$
F(y)=1-e^{-y / \theta_{1}} \text { and } G(w)=1-e^{-w / \theta_{2}}, y>0, w>0 ; \theta_{i}>0, i=1,2 .
$$

Assuming $T_{i}=x_{r_{i}}-x_{r_{i-1}}, i=2, \ldots, k$ and substituting Eq. (2) in Eq. (1) the likelihood function becomes

$$
\begin{aligned}
L=L(\theta \mid X)= & \frac{C}{\theta_{1}^{m_{k}}} \theta_{2}^{n_{k}}\left(1-e^{-\frac{x}{\theta_{1}}}\right)^{a_{1}} \exp \left(-\frac{u_{k}}{\theta_{1}}\right) \prod_{i=2}^{k}\left[1-e^{-T_{\frac{i}{\theta_{1}}}}\right]^{a_{i}}\left(1-e^{-\frac{x_{1_{1}}}{\theta_{2}}}\right)^{b_{1}} \exp \left(-\frac{v_{k}}{\theta_{2}}\right) \\
& \times \prod_{i=2}^{k}\left[1-e^{-T_{i}} \frac{i}{\theta_{2}}\right]^{b_{i}}
\end{aligned}
$$

where

$$
\theta=\left(\theta_{1}, \theta_{2}\right), u_{k}=\sum_{i=2}^{k} a_{i} x_{r_{i-1}}+\sum_{i=1}^{k} z_{i} x_{r_{i}}+a_{k+1} x_{r_{k}}
$$

and

$$
v_{k}=\sum_{i=2}^{k} b_{i} x_{r_{i-1}}+\sum_{i=1}^{k}\left(1-z_{i}\right) x_{r_{i}}+b_{k+1} x_{r_{k}}
$$

\section{Maximum likelihood estimate (MLE) and asymptotic standard error}

The MLEs $\hat{\theta}_{1}$ and $\hat{\theta}_{2}$ of the parameters $\theta_{1}$ and $\theta_{2}$ are obtained by maximizing Eq. (3). To maximize the likelihood function in Eq. (3) we derive the likelihood equations

$$
\frac{\partial \log L}{\partial \theta_{1}}=0 \text { and } \frac{\partial \log L}{\partial \theta_{2}}=0 .
$$

Solving the above equations we get MLEs of mean life times $\theta_{1}$ and $\theta_{2}$.The maximum likelihood method does not admit explicit solutions, but we have two equations as

$$
m_{k} \theta_{1}=u_{k}-\frac{a_{1} x}{{ }_{r_{1}} e^{-\left(x_{r_{1}} / \theta_{1}\right)}}-\sum_{i=2}^{k}\left\{\frac{a_{i} T_{i} e^{-\left(T_{i} / \theta_{1}\right)}}{1-e^{-\left(x_{r_{1}} / \theta_{1}\right)}}\right\}
$$

and 


$$
n_{k} \theta_{2}=v_{k}-\frac{b_{1} x_{r_{1} e^{-\left(x_{r_{1}} / \theta_{2}\right)}}}{1-e^{-\left(x_{r_{1}} / \theta_{2}\right)}}-\sum_{i=2}^{k}\left\{\frac{b_{i} T_{i} e^{-\left(T_{i} / \theta_{2}\right)}}{1-e^{-\left(T_{i} / \theta_{2}\right)}}\right\}
$$

Using any method of iteration, the Eqs. (4) and (5) can be solved for $\theta_{1}$ and $\theta_{2}$. The solutions of the two equations give us the MLEs $\hat{\theta}_{1}$ and $\hat{\theta}_{2}$ of the parameters $\theta_{1}$ and $\theta_{2}$ respectively.

Let $I\left(\theta_{1}, \theta_{2}\right)=I_{i, j}\left(\theta_{i}, \theta_{j}\right) i, j=1,2$ denote the Fisher information matrix of the parameters $\theta_{1}$ and $\theta_{2}$, whereand consequently the observed Fisher information matrix is given by

$$
\begin{aligned}
& I_{i, j}\left(\theta_{i}, \theta_{j}\right)=-E\left(\frac{\partial^{2} \log L}{\partial \theta_{i} \partial \theta_{j}}\right) \\
& \hat{I}\left(\theta_{1}, \theta_{2}\right)=\left(\begin{array}{cc}
-\frac{\partial^{2} \log L}{\partial \theta_{1}^{2}} & 0 \\
0 & -\frac{\partial^{2} \log L}{\partial \theta_{2}^{2}}
\end{array}\right)_{\left(\theta_{1}, \theta_{2}\right)=\left(\hat{\theta}_{1}, \hat{\theta}_{2}\right)}
\end{aligned}
$$

where

$$
\begin{aligned}
\frac{\partial^{2} \log L}{\partial \theta_{1}^{2}}= & \frac{2}{\theta_{1}^{3}}\left\{\frac{a_{1} x_{r_{1}} e^{-\left(x_{r_{1}} / \theta_{1}\right)}}{1-e^{-\left(x_{r_{1}} / \theta_{1}\right)}}\right\}-\frac{a_{1} x_{r_{1}}^{2}}{\theta_{1}^{4}} \frac{e^{-\left(x_{r_{1}} / \theta_{1}\right)}}{\left(1-e^{-\left(x_{r_{1}} / \theta_{1}\right)}\right)^{2}}-\frac{2 u_{k}}{\theta_{1}^{3}}+\frac{m_{k}}{\theta_{1}^{2}}+\frac{2}{\theta_{1}^{3}} \sum_{i=2}^{k} \frac{a_{i} T_{i} e^{-\left(T_{i} / \theta_{1}\right)}}{1-e^{-\left(T_{i} / \theta_{1}\right)}} \\
& -\frac{1}{\theta_{1}^{4}} \sum_{i=2}^{k} \frac{a_{i} T_{i}^{2} e^{-\left(T_{i} / \theta_{1}\right)}}{\left(1-e^{-\left(T_{i} / \theta_{1}\right)}\right)^{2}}
\end{aligned}
$$

and

$$
\begin{aligned}
\frac{\partial^{2} \log L}{\partial \theta_{2}^{2}}= & \frac{2}{\theta_{2}^{3}}\left\{\frac{b_{1} x_{r_{1}} e^{-\left(x_{r_{1}} / \theta_{2}\right)}}{1-e^{-\left(x_{r_{1}} / \theta_{2}\right)}}\right\}-\frac{b_{1} x_{r_{1}}^{2}}{\theta_{2}^{4}} \frac{e^{-\left(x_{r_{1}} / \theta_{2}\right)}}{\left(1-e^{-\left(x_{r_{1}} / \theta_{2}\right)}\right)^{2}}-\frac{2 v_{k}}{\theta_{2}^{3}}+\frac{n_{k}}{\theta_{2}^{2}}+\frac{2}{\theta_{2}^{3}} \sum_{i=2}^{k} \frac{b_{i} T_{i} e^{-\left(T_{i} / \theta_{2}\right)}}{1-e^{-\left(T_{i} / \theta_{2}\right)}} \\
& -\frac{1}{\theta_{2}^{4}} \sum_{i=2}^{k} \frac{b_{i} T_{i}^{2} e^{-\left(T_{i} / \theta_{2}\right)}}{\left(1-e^{-\left(T_{i} / \theta_{2}\right)}\right)^{2}}
\end{aligned}
$$

Hence asymptotic standard errors (ASEs) of MLEs are obtained by

$$
\operatorname{ASE}\left(\hat{\theta}_{1}\right)=\sqrt{\frac{-1}{E\left(\frac{\partial^{2} \log L}{\partial \theta_{1}^{2}}\right)}} \text { and } \operatorname{ASE}\left(\hat{\theta}_{2}\right)=\sqrt{\frac{-1}{E\left(\frac{\partial^{2} \log L}{\partial \theta_{2}^{2}}\right)}}
$$

Then, by using the asymptotic normality of the MLEs, we can express the asymptotic $(1-\alpha) 100 \%$ confidence intervals for $\theta_{1}$ and $\theta_{2}$ as

$$
\hat{\theta}_{1} \pm Z_{\alpha / 2} \operatorname{ASE}\left(\hat{\theta}_{1}\right) \text { and } \hat{\theta}_{2} \pm Z_{\alpha / 2} \operatorname{ASE}\left(\hat{\theta}_{2}\right)
$$

where $Z_{\alpha / 2}$ denotes the upper $\alpha / 2$ percentage point of the standard normal distribution.

MLEs of Reliabilities at time $t$ of the product of type-A and type-B are respectively given by 


$$
\widehat{R_{\mathrm{A}}}(t)=e^{-t / \hat{\theta}_{1}} \text { and } \widehat{R_{\mathrm{B}}}(t)=e^{-t / \hat{\theta}_{2}}
$$

The ASE of MLE of reliability at time $t$ for the product A is calculated as

$$
\operatorname{ASE}_{\mathrm{A}}(t)=\left.\sqrt{\frac{\mathrm{d} R_{\mathrm{A}}(t)}{\mathrm{d} \theta_{1}} V\left(\hat{\theta}_{1}\right)}\right|_{\theta=\hat{\theta}_{1}}
$$

Similarly the asymptotic standard error of MLE of reliability at time $t$ for the product B can be calculated.

\section{Influence measure}

In this section we have considered the influence of individual observations on maximum likelihood estimates. We have used the method considered by Poon and Tang [22].

Let $L(\theta)$ be the likelihood function of $\theta$ given in Eq. (1). Define the case weight perturbation $w=\left(w_{1}, w_{2}, \ldots, w_{N}\right)^{\prime}$ and corresponding perturbed log-likelihood function will be

$$
\begin{aligned}
\log L(\theta \mid w)= & \sum_{i=1}^{a_{1}} w_{i} \log \left(F\left(x_{r_{1}}, \theta_{1}\right)\right)+\sum_{i=a_{1}+1}^{r_{1}-1} w_{i} \log \left(G\left(x_{r_{1}}, \theta_{2}\right)\right)+\sum_{i=1}^{k} w_{r_{i}} z_{i} \log \left(f\left(x_{r_{1}}, \theta_{1}\right)\right) \\
& +\sum_{i=1}^{k} w_{r_{i}}\left(1-z_{i}\right) \log \left(g\left(x_{r_{1}}, \theta_{2}\right)\right)+\sum_{j=2}^{k} \sum_{i=r_{j-1}+1}^{a_{j}+r_{j-1}} w_{i} \log \left[F\left(x_{r_{j}}, \theta_{1}\right)-F\left(x_{r_{j-1}}, \theta_{1}\right)\right] \\
& +\sum_{j=2}^{k} \sum_{i=a_{j}+r_{j-1}+1}^{r_{j}-1} w_{i} \log \left[G\left(x_{r_{j}}, \theta_{2}\right)-G\left(x_{r_{j-1}}, \theta_{2}\right)\right] \\
& +\sum_{i=r_{k}+1}^{a_{k+1}+r_{k}} w_{i} \log \left[1-F\left(x_{r_{k}}, \theta_{1}\right)\right]+\sum_{i=a_{k+1}+r_{k}+1}^{N} w_{i} \log \left[1-G\left(x_{r_{k}}, \theta_{2}\right)\right]
\end{aligned}
$$

Using Eq. (2) in Eq. (13) it can be further simplified as

$$
\begin{aligned}
\log L(\theta \mid w)= & \sum_{i=1}^{a_{1}} w_{i} \log \left(1-\exp \left(-\frac{x_{r_{1}}}{\theta_{1}}\right)\right)+\sum_{i=a_{1}+1}^{r_{1}-1} w_{i} \log \left(1-\exp \left(-\frac{x_{r_{1}}}{\theta_{2}}\right)\right) \\
& -\left(\frac{1}{\theta_{1}}\right)\left\{\sum_{i=1}^{k} w_{r_{i}} z_{i} x_{r_{i}}+\sum_{j=2}^{k} \sum_{i=r_{j-1}+1}^{a_{j}+r_{j-1}} w_{i} x_{r_{j-1}}+\sum_{i=r_{k}+1}^{a_{k+1}+r_{k}} w_{i} x_{r_{k}}\right\} \\
& -\left(\frac{1}{\theta_{2}}\right)\left\{\sum_{i=1}^{k} w_{r_{i}}\left(1-z_{i}\right) x_{r_{i}}+\sum_{j=2}^{k} \sum_{i=a_{j}+r_{j-1}+1}^{r_{j}-1} w_{i} x_{r_{j-1}}+\sum_{i=a_{k+1}+r_{k}+1}^{N} w_{i} x_{r_{k}}\right\} \\
& -\sum_{i=1}^{k} w_{r_{i}} z_{i} \log \theta_{1}+\sum_{j=2}^{k} \sum_{i=r_{j-1}+1}^{a_{j}+r_{j-1}} w_{i} \log \left(1-\exp \left(-\frac{T_{j}}{\theta_{1}}\right)\right) \\
& -\sum_{i=1}^{k} w_{r_{i}}\left(1-z_{i}\right) \log \theta_{2}+\sum_{j=2}^{k} \sum_{i=a_{j}+r_{j-1}+1}^{r_{j}-1} w_{i} \log \left(1-\exp \left(-\frac{T_{j}}{\theta_{2}}\right)\right)
\end{aligned}
$$

If $w=w_{0}=(1,1, \ldots, 1)^{\prime}$, from Eq. (14) we see that $\log L\left(\theta \mid w_{0}\right)=\log L(\theta)$. If $i$ th observation is deleted i.e. $w_{i}=0$ and if such deletion leads to very different MLE of 
$\theta=\left(\theta_{1}, \theta_{2}\right)$, then it leads to influence of $i$-th observation. In a similar manner if a small perturbation of $w_{i}$ from $w_{i}=1$ leads to a very different $\operatorname{MLE}$ of $\theta=\left(\theta_{1}, \theta_{2}\right)$, it will be evidence of influence of $i$-th observation. Thus, if $\log L(\theta \mid w)$ becomes maximum under $\hat{\theta}_{w}$, then the change of $\hat{\theta}_{w}$, as a function of w reveals the information about how influential an individual observation is. Cook [23] proposed the displacement $D(w)$ as

$$
D(w)=\log L\left(\hat{\theta} \mid w_{0}\right)-\log L\left(\hat{\theta}_{w} \mid w_{0}\right)
$$

The directions giving large change of $D(w)$ at $w_{0}$ are of interest. Cook [23] proposed the following straightforward computation method of such a direction as described below:

$$
\text { Let } H=\frac{\partial^{2} \log L(\theta)}{\partial \theta_{1} \partial \theta_{2}} \mid\left(\theta_{1}, \theta_{2}\right)=\left(\hat{\theta}_{1}, \hat{\theta}_{2}\right)
$$

which is a $2 \times 2$ matrix.

Define

$$
A=\left(\frac{\partial^{2} \log L(\theta \mid w)}{\partial\left(\theta_{1}, \theta_{2}\right) \partial w}\right)_{\left(\theta_{1}, \theta_{2}\right)=\left(\hat{\theta}_{1}, \hat{\theta}_{2}\right), w=w_{0}}
$$

which is a $2 \times N$ matrix, $N=m+n$ is a total sample size.

The $(1, j)$ th element of the matrix $A$ is given by

$$
\frac{\partial^{2} \log L(\theta \mid w)}{\partial \theta_{1} \partial w j} \mid \theta_{1}=\hat{\theta}_{1}, w=w_{0_{j}}
$$

and $(2, j)$ th element as

$$
\frac{\partial^{2} \log L(\theta \mid w)}{\partial \theta_{2} \partial w j} \mid \theta_{2}=\hat{\theta}_{2}, w=w_{0_{j}}
$$

Using the Eqs. (7) and (8) the matrix $H$ can be computed at MLE of the parameters.

Then matrix $\Lambda$ is defined as

$$
\Lambda=-A^{\prime}(H)^{-1} A
$$

Poon and Poon [24] introduced the basic perturbation direction $\left(\mathrm{pd}_{i}\right)$ with the help of diagonal elements and trace value of the matrix $\Lambda$.

$$
\operatorname{pd}_{i}=\frac{\Lambda_{i i}}{\sqrt{\operatorname{trace}\left(\Lambda^{2}\right)}}, \quad i=1,2, \ldots N
$$

and suggest that the observations with large $\mathrm{pd}_{i}$ values as influential. From Eqs. (16) to (17), influence measures can be computed with given density function $f\left(x_{i}, \theta\right)$. To identify the large values of $\mathrm{pd}_{i}$, Poon and Poon [24] introduced the reference constant $(c)$ as

$$
c=\frac{2 \operatorname{trace}(\Lambda)}{N \sqrt{\operatorname{trace}\left(\Lambda^{2}\right)}}
$$

which can be used to identify the observations having large $\mathrm{pd}_{i}$ values. 


\section{Bayes estimation}

In this section we consider the Bayes estimates of mean life time and reliability of the products of type-A and type-B. To obtain Bayes estimate of the parameter $\theta$ of the distribution, we should decide prior distribution of $\theta$. Prior distribution can be determined by analyst's pre-data understanding/knowledge/belief about $\theta$. Usually the parametric form of the prior is chosen such that the posterior distribution of $\theta$ be of the same form i.e. belongs to the same family of the prior distributions. Use of such a prior is mostly for mathematical and computational convenience in practice. Usually the Bayes estimate of the parameter $\theta$ fall somewhere between the prior and likelihood estimates. Thus Bayes estimate of the parameter depends on the initial beliefs about the parameter $\theta$.

Unfortunately, when both the parameters $\theta_{1}$ and $\theta_{2}$ are unknown then there does not exist any natural conjugate priors. In this article, similarly as in Kundu and Pradhan [25], we use the inverse gamma priors for the parameters $\theta_{1}$ and $\theta_{2}$.

The inverse gamma priors IG $\left(c_{1}, d_{1}\right)$ and $\operatorname{IG}\left(c_{2}, d_{2}\right)$ for $\theta_{1}$ and $\theta_{2}$ respectively defined as

$$
\begin{aligned}
& \pi_{1}\left(\theta_{1}\right)=\frac{e^{-d_{1} / \theta_{1}} d_{1}^{c_{1}}}{\theta_{1}^{c_{1}+1} \Gamma c_{1}}=\operatorname{IG}\left(c_{1}, d_{1}\right) \text { and } \pi_{2}\left(\theta_{2}\right)=\frac{e^{-d_{2} / \theta_{2}} d_{2}^{c_{2}}}{\theta_{2}^{c_{2}+1} \Gamma c_{2}}=\operatorname{IG}\left(c_{2}, d_{2}\right), \\
& \theta_{i}>0, a_{i}>0, b_{i}>0, i=1,2 .
\end{aligned}
$$

On the basis of the likelihood function in Eq. (3) and above independent inverse gamma prior distributions, the joint posterior density function of $\theta_{1}$ and $\theta_{2}$ can be constructed as

$$
\begin{aligned}
& h\left(\theta_{1}, \theta_{2} \mid \underline{x}\right)=L \pi_{1}\left(\theta_{1}\right) \pi_{2}\left(\theta_{2}\right) \\
& \propto \frac{e^{-\frac{\left(u_{k}+d_{1}\right)}{\theta_{1}}}\left(u_{k}+d_{1}\right)^{m_{k}+c_{1}}}{\theta_{1}^{m_{k}+c_{1}+1} \Gamma\left(m_{k}+c_{1}\right)} \frac{e^{-\frac{\left(v_{k}+d_{2}\right)}{\theta_{2}}}\left(v_{k}+d_{2}\right)^{n_{k}+c_{2}}}{\theta_{2}^{n_{k}+c_{2}+1} \Gamma\left(n_{k}+c_{2}\right)} \frac{\left(1-e^{-\left(\frac{x_{1}}{\theta_{1}}\right)}\right)^{a_{1}}\left(1-e^{-\left(\frac{x_{r_{1}}}{\theta_{2}}\right)}\right)^{b_{1}}}{\left(u_{k}+d_{1}\right)^{m_{k}+c_{1}}\left(v_{k}+d_{2}\right)^{n_{k}+c_{2}}} \\
& \left.\quad \times \prod_{i=2}^{k}\left[1-e^{-T \frac{i}{\theta_{1}}}\right]^{a_{i}} \prod_{i=2}^{k}\left[1-e^{-T_{i}}\right]^{\theta_{2}}\right]^{b_{i}} \\
& \propto \operatorname{IG}\left(m_{k}+c_{1}, u_{k}+d_{1}\right) \operatorname{IG}\left(n_{k}+c_{2}, v_{k}+d_{2}\right) h_{3}\left(\theta_{1}, \theta_{2} \mid \underline{x}\right)
\end{aligned}
$$

where

$$
h_{3}\left(\theta_{1}, \theta_{2} \mid \underline{x}\right)=\frac{\left(1-e^{-\left(\frac{x_{r_{1}}}{\theta_{1}}\right)}\right)^{a_{1}}\left(1-e^{-\left(\frac{x_{r_{1}}}{\theta_{2}}\right)}\right)^{b_{1}}}{\left(u_{k}+d_{1}\right)^{m_{k}+c_{1}}\left(\nu_{k}+d_{2}\right)^{n_{k}+c_{2}}} \prod_{i=2}^{k}\left[1-e^{-T_{\frac{i}{\theta_{1}}}}\right]^{a_{i}} \prod_{i=2}^{k}\left[1-e^{-T_{\frac{i}{\theta_{2}}}}\right]^{b_{i}}
$$

From the expression of the posterior distribution given in (14) it is quite difficult to obtain Bayes estimates of the parameters in closed form, so we use approximation method to evaluate them. There are several approximation methods to obtain Bayes estimates of the parameters. Here, we use importance sampling method proposed by Kundu and Pradhan [25]. The importance sampling method can be used to derive estimates of parameters.

Using importance sampling approach Bayes estimates of $\theta_{1}$ and $\theta_{2}$ can be obtained as follows: 
Step 1: Generate $\theta_{1}$ from IG $\left(m_{k}+c_{1}, u_{k}+d_{1}\right) \sim \operatorname{Inverse~gamma~}\left(m_{k}+c_{1}, u_{k}+d_{1}\right)$

Step 2: Generate $\theta_{2}$ from IG $\left(n_{k}+c_{2}, v_{k}+d_{2}\right) \sim$ Inverse gamma $\left(n_{k}+c_{2}, v_{k}+d_{2}\right)$

Step 3: Repeat Steps 1 and $2 \mathrm{~N}$ times to obtain $\left(\theta_{11}, \theta_{21}\right), \ldots,\left(\theta_{1 N}, \theta_{2 N}\right)$

Step 4: The Bayes estimate $\hat{\varepsilon}_{\mathbf{B}}$ of $\varepsilon\left(\theta_{1}, \theta_{2}\right)$, any function of $\theta_{1}$ and $\theta_{2}$ under the squarederror loss function can then be approximated as

$$
\hat{\varepsilon}_{\mathrm{B}}=\frac{\sum_{i=1}^{N} \varepsilon\left(\theta_{1 i}, \theta_{2 i}\right) h_{3}\left(\theta_{1 i}, \theta_{i 2} \mid \underline{x}\right)}{\sum_{i=1}^{N} h_{3}\left(\theta_{1 i}, \theta_{2 i} \mid \underline{x}\right)}
$$

The Bayes estimate of $\theta_{1}$ is obtained by considering $\varepsilon\left(\theta_{1}, \theta_{2}\right)=\theta_{1}$ in the above computation.

Similarly, the Bayes estimate of $\theta_{2}$ can be computed. The Bayes estimates of the reliabilities at time $t$ of the two types of products can be obtained by replacing the function $\varepsilon\left(\theta_{1}, \theta_{2}\right)$ by an expression of reliability function given in (11). Some further applications of this method can also be found in Kundu and Pradhan [25] and Rastogi and Tripathi [26].

\section{Results and discussion}

In this section we perform the analysis of a real data set to illustrate how the proposed methods work in practice. We have used the following data set proposed by Proschan [11]. Proschan [11] presented data on intervals between failures (in hours) of the air conditioning system of a fleet of 13 Boeing 720 jet airplanes. After analyzing the data, he observed that the failure distribution of the air-conditioning system for each of the planes was well approximated by exponential distributions. These data sets were used by Rasouli and Balakrishnan [7] and Mondal and Kundu [27].

For the purpose of illustration, we chose here the planes "7914" and "7913," and we assume the failure times of air-conditioning systems in the two planes follow exponential life time model with means $\theta_{1}>0$ and $\theta_{2}>0$ respectively. The samples of sizes $m=24$ and $n=27$ for plane 7914 and 7913 are considered respectively. The corresponding failure time data are presented in Table 1.

Here we call the air-conditioning systems of plane 7914 as product of type-A and of plane 7913 as product of type-B. As an example, we have constructed two jointly multiply type-II censored samples with $k=9$ and $k=6$ from the above data to exemplify the results obtained in the earlier sections, which are shown in the Tables 2 and 3 respectively.

Using the Eqs. (4), (5) and (9) the MLEs and their asymptotic standard errors are calculated and presented in the Table 4. The results are obtained in case of joint multiply type-II as well as for usual joint type-II censored samples.

Table 1 Failure times of air-conditioning systems in two airplanes

\begin{tabular}{ll}
\hline Plane & Failure times \\
\hline 7914 & $3,5,5,13,14,15,22,22,23,30,36,39,44,46,50,72,79,88,97,102,139,188,197,210$ \\
7913 & $1,4,11,16,18,18,18,24,31,39,46,51,54,63,68,77,80,82,97,106,111,141,142$, \\
& $163,191,206,216$
\end{tabular}


Table 2 Joint multiply type-II censoring scheme with $k=9$

\begin{tabular}{lrrrrrrrrrr}
\hline $\boldsymbol{i}$ & $\mathbf{1}$ & $\mathbf{2}$ & $\mathbf{3}$ & $\mathbf{4}$ & $\mathbf{5}$ & $\mathbf{6}$ & $\mathbf{7}$ & $\mathbf{8}$ & $\mathbf{9}$ \\
\hline$r_{i}$ & 3 & 8 & 14 & 22 & 27 & 31 & 37 & 40 & 43 \\
$X_{r_{i}}$ & 4 & 14 & 22 & 39 & 51 & 72 & 97 & 106 & 141 \\
$Z$ & 0 & 1 & 1 & 0 & 0 & 1 & 1 & 0 & 0 & \\
$a_{i}$ & 1 & 3 & 1 & 5 & 3 & 0 & 2 & 1 & 1 & 3 \\
$b_{i}$ & 1 & 1 & 4 & 2 & 1 & 3 & 3 & 1 & 1 & 5 \\
$R_{i}$ & 2 & 4 & 5 & 7 & 4 & 3 & 5 & 2 & 2 & 8 \\
$T_{i}$ & - & 10 & 8 & 17 & 12 & 21 & 25 & 9 & 35 & \\
\hline
\end{tabular}

Table 3 Joint multiply type-II censoring scheme with $k=6$

\begin{tabular}{lrrrrrrr}
\hline $\boldsymbol{i}$ & $\mathbf{1}$ & $\mathbf{2}$ & $\mathbf{3}$ & $\mathbf{4}$ & $\mathbf{5}$ & $\mathbf{6}$ \\
\hline$r_{i}$ & 3 & 8 & 14 & 22 & 27 & 31 \\
$X_{r_{i}}$ & 4 & 14 & 22 & 39 & 51 & 72 \\
$Z$ & 0 & 1 & 1 & 0 & 0 & 1 & \\
$a_{i}$ & 1 & 3 & 1 & 5 & 3 & 0 & 8 \\
$b_{i}$ & 1 & 1 & 4 & 2 & 1 & 3 & 12 \\
$R_{i}$ & 2 & 4 & 5 & 7 & 4 & 3 & 20 \\
$T_{i}$ & - & 10 & 8 & 17 & 12 & 21 & \\
\hline
\end{tabular}

Table 4 MLE and ASE

\begin{tabular}{|c|c|c|c|c|c|}
\hline \multirow[t]{2}{*}{ Censoring scheme } & \multirow[t]{2}{*}{$k$} & \multicolumn{2}{|l|}{ MLE } & \multicolumn{2}{|l|}{ ASE } \\
\hline & & $\hat{\theta}_{1}$ & $\hat{\theta}_{2}$ & $\operatorname{ASE}\left(\hat{\theta}_{1}\right)$ & $\operatorname{ASE}\left(\hat{\theta}_{2}\right)$ \\
\hline \multirow[t]{2}{*}{ Joint multiply type-II } & 9 & 59.51328 & 86.02658 & 12.08575 & 9.25042 \\
\hline & 6 & 63.47124 & 88.61522 & 12.96159 & 22.89991 \\
\hline \multirow[t]{2}{*}{ Joint type-II } & 9 & 54.16666 & 125.3333 & 22.11366 & 72.36123 \\
\hline & 6 & 81.33333 & 93.33333 & 46.95782 & 53.88602 \\
\hline
\end{tabular}

MLEs of reliabilities at time $t=100$ for both the types of products along with their asymptotic standard errors are calculated using the Eqs. (11) and (12) and shown in the Table 5.

Estimation of $\theta_{1}$ is quite stable over different choice of $k$, the estimation of $\theta_{2}$ seems to be quite sensitive to the joint multiply type II censoring scheme employed when $k$ is small. This effect also observed in reliability of the products.

When two populations are not identical and consider smaller samples from them, then ordinary joint type-II censoring scheme resulting in poor inference for mean of both the populations. From Table 4, we observed that asymptotic standard errors for each of the product $\mathrm{A}$ and product $\mathrm{B}$ are smaller in case of joint multiply type-II censoring scheme than that of under joint type-II censoring scheme. Which reveals the advantage of joint multiply type-II censoring over the joint type-II censoring.

Now we calculate influence measure for the joint multiply type-II censored sample with $k=6$, with the data given in Table 3 . 
Table 5 Reliability and ASE

\begin{tabular}{lllllll}
\hline Censoring scheme & $\boldsymbol{k}$ & \multicolumn{2}{l}{ Reliability at $\boldsymbol{t}=\mathbf{1 0 0}$} & & \multicolumn{2}{l}{ ASE } \\
\cline { 3 - 4 } & & type-A & type-B & & type-A & type-B \\
\hline Joint multiply type-II & 9 & 0.18632 & 0.31273 & & 0.00526 & 0.00422 \\
& 6 & 0.20690 & 0.32352 & & 0.00513 & 0.00412 \\
Joint type-II & 9 & 0.15784 & 0.45029 & & 0.00538 & 0.00287 \\
& 6 & 0.29244 & 0.34252 & & 0.00442 & 0.00393 \\
\hline
\end{tabular}

In our example, we have total 51 products including both the types $\mathrm{A}$ and type $\mathrm{B}$, so the order of matrix $A$ will be $2 \times 51$.

The 51 elements $a_{1 w_{j}}, j=1,2, \ldots, 51$ of the first row of matrix $A$ can be calculated using Eq. (18) as

$$
\begin{aligned}
& a_{1 w_{j}}=\frac{-\frac{x_{r_{1}}}{\theta_{1}^{2}} e^{-\frac{x_{r_{1}}}{\theta_{1}}}}{1-e^{-\frac{x_{r_{1}}}{\theta_{1}}}}, \quad \text { for } j=1 \\
& a_{1 w_{r_{j}}}=\frac{Z_{j} x_{r_{j}}}{\theta_{1}^{2}}-\frac{Z_{j}}{\theta_{1}}, \quad \text { for } j=1,2, \ldots, 6 \\
& a_{1 w_{j}}=\frac{-\frac{T_{2}}{\theta_{1}^{2}} e^{-\frac{T_{2}}{\theta_{1}}}}{1-e^{-\frac{T_{2}}{\theta_{1}}}}+\frac{x_{3}}{\theta_{1}^{2}}, \text { for } j=4,5,6 \\
& a_{1 w_{j}}=\frac{-\frac{T_{3}}{\theta_{1}^{2}} e^{-\frac{T_{3}}{\theta_{1}}}}{1-e^{-\frac{T_{3}}{\theta_{1}}}}+\frac{x_{8}}{\theta_{1}^{2}}, \quad \text { for } j=9 \\
& a_{1 w_{j}}=\frac{-\frac{T_{4}}{\theta_{1}^{2}} e^{-\frac{T_{4}}{\theta_{1}}}}{1-e^{-\frac{T_{4}}{\theta_{1}}}}+\frac{x_{14}}{\theta_{1}^{2}}, \quad \text { for } j=15 \text { to } 19 \\
& a_{1 w_{j}}=\frac{-\frac{T_{5}}{\theta_{1}^{2}} e^{-\frac{T_{5}}{\theta_{1}}}}{1-e^{-\frac{T_{5}}{\theta_{1}}}}+\frac{x_{22}}{\theta_{1}^{2}}, \quad \text { for } 23 \text { to } 25 \\
& a_{1 w_{j}}=\frac{-\frac{T_{6}}{\theta_{1}^{2}} e^{-\frac{T_{6}}{\theta_{1}}}}{1-e^{-\frac{T_{6}}{\theta_{1}}}}+\frac{x_{27}}{\theta_{1}^{2}}, \text { for } 28 \\
& a_{1 w_{j}}=\frac{x_{31}}{\theta_{1}^{2}}, \quad \text { for } j=32 \text { to } 39
\end{aligned}
$$

and 
$a_{1 w_{j}}=0, \quad$ for all remaining $j$

Similarly the 51 elements $a_{2 w_{j}}, j=1,2, \ldots, 51$ of the second row of the matrix $A$ are calculated using Eq. (19) by replacing $\theta_{1}$ by $\theta_{2}$ in the expressions of the elements of the first row of matrix $A$ given above. Replacing the MLEs $\hat{\theta}_{1}$ and $\hat{\theta}_{2}$ in place of $\theta_{1}$ and $\theta_{2}$, and substituting the values in vector $\mathrm{w}_{0}$ the numeric values of the elements of the matrix $A$ can be obtained.

The matrix $H^{-1}$ can be obtained from Eq. (16) at MLEs as

$$
H^{-1}=\left[\begin{array}{cc}
-168.002810 & 0 \\
0 & -524.405878
\end{array}\right]
$$

Hence the elements of matrix $\Lambda$ can be calculated using Eq. (20). The basic perturbation direction values $\left(\mathrm{pd}_{i}\right)$ from Eq. (21) comes out as given in Table 6.

From Eq. (22) the reference constant $c$ is calculated and comes out 0.05456 .

To identify the observations which have greater effect on MLEs, we find out the $\mathrm{pd}_{i}$ values. The plot of $\mathrm{pd}_{i}$ versus $i$, with reference line at constant $c$ is given in Fig. 1. From the plot we observe that no observations have $\mathrm{pd}_{i}$ values more than the reference constant $c$. Hence none of the observations influence MLEs in a disproportionate way.

Bayes estimates of mean life times and reliabilities of the products are presented in Tables 7 and 8 respectively based on the "Methods" section in case of $k=9$ and 6 for different choice of prior parameters $c_{1}, d_{1}, c_{2}$ and $d_{2}$.There are many ways of determining the values of prior parameters. The values for different prior parameters can be chosen by utilizing the prior information from the past data. Here we have adopted the method used by Sultana et al. [28]. Suppose that $N$ numbers of past data are available from the given population. Let $\hat{\theta}_{1_{j}}$ and $\hat{\theta}_{2_{j}}, j=1,2, \ldots N$ denote the corresponding MLEs of unknown parameters $\theta_{1}$ and $\theta_{2}$. The selection of prior parameters can be made by equating mean and variance of $\hat{\theta}_{1_{j}}$ and $\hat{\theta}_{2_{j}}$ with the corresponding mean and variance of the prior distributions.

From the above table, we observe that increase in the value of $c_{1}\left(c_{2}\right)$ Bayes estimate of $\theta_{1}\left(\theta_{2}\right)$ are sensitive and decreases in both the cases of censoring schemes, but Bayes estimate of $\theta_{1}\left(\theta_{2}\right)$ remains almost stable under changes in the value of $d_{1}\left(d_{2}\right)$. Under the

Table 6 Values of $\mathrm{pd}_{i}$

\begin{tabular}{llllllllll}
\hline $\boldsymbol{i}$ & $\mathbf{p d}_{\boldsymbol{i}}$ & $\boldsymbol{i}$ & $\mathbf{p d}_{\boldsymbol{i}}$ & $\boldsymbol{i}$ & $\mathbf{p d}_{\boldsymbol{i}}$ & $\boldsymbol{i}$ & $\mathbf{p d}_{\boldsymbol{i}}$ & $\boldsymbol{i}$ & $\mathbf{p d}_{\boldsymbol{i}}$ \\
\hline 1 & 0.032053 & 11 & 0.034785 & 21 & 0.023740 & 31 & 0.000617 & 41 & 0.036101 \\
2 & 0.052264 & 12 & 0.034785 & 22 & 0.017143 & 32 & 0.043944 & 42 & 0.036101 \\
3 & 0.049860 & 13 & 0.034785 & 23 & 0.002952 & 33 & 0.043944 & 43 & 0.036101 \\
4 & 0.025273 & 14 & 0.014579 & 24 & 0.002952 & 34 & 0.043944 & 44 & 0.036101 \\
5 & 0.025273 & 15 & 0.009428 & 25 & 0.002952 & 35 & 0.043944 & 45 & 0.036101 \\
6 & 0.025273 & 16 & 0.009428 & 26 & 0.013330 & 36 & 0.043944 & 46 & 0.036101 \\
7 & 0.044246 & 17 & 0.009428 & 27 & 0.009853 & 37 & 0.043944 & 47 & 0.036101 \\
8 & 0.020746 & 18 & 0.009428 & 28 & 0.005278 & 38 & 0.043944 & 48 & 0.036101 \\
9 & 0.017592 & 19 & 0.009428 & 29 & 0.005278 & 39 & 0.043944 & 49 & 0.036101 \\
10 & 0.034785 & 20 & 0.023740 & 30 & 0.005278 & 40 & 0.036101 & 50 & 0.036101 \\
& & & & & & & & 51 & 0.036101 \\
\hline
\end{tabular}




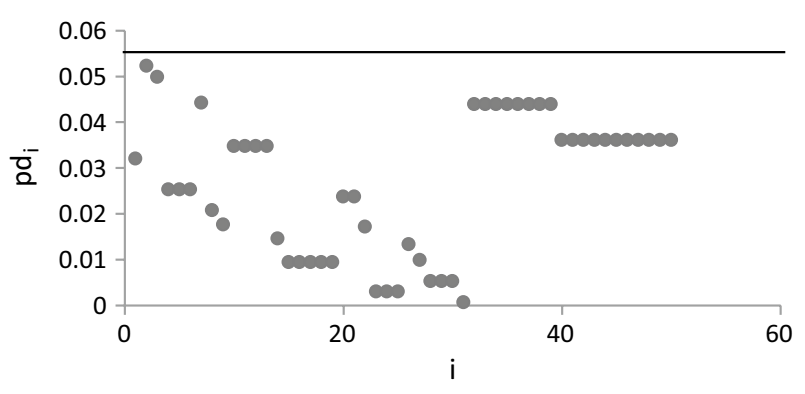

Fig. 1 Values of $\mathrm{pd}_{i}$ versus $i$ and reference line at value $c=0.05456$

Table 7 Bayes estimates of mean life times

\begin{tabular}{|c|c|c|c|c|c|c|c|}
\hline \multirow[t]{2}{*}{$c_{1}$} & \multirow[t]{2}{*}{$d_{1}$} & \multirow[t]{2}{*}{$c_{2}$} & \multirow[t]{2}{*}{$d_{2}$} & \multicolumn{2}{|l|}{$k=9$} & \multicolumn{2}{|l|}{$k=6$} \\
\hline & & & & $\hat{\theta}_{1}$ & $\hat{\theta}_{2}$ & $\hat{\theta}_{1}$ & $\hat{\theta}_{2}$ \\
\hline 18 & 999 & 40 & 2861 & 73.7492 & 80.9237 & 65.1143 & 74.6591 \\
\hline 15 & 999 & 40 & 2861 & 83.4231 & 81.0132 & 73.9114 & 74.6439 \\
\hline 21 & 999 & 40 & 2861 & 66.1775 & 80.8727 & 58.2935 & 74.7057 \\
\hline 18 & 1010 & 40 & 2861 & 74.1121 & 80.9243 & 65.4957 & 74.6591 \\
\hline 18 & 990 & 40 & 2861 & 73.4522 & 80.9232 & 64.8022 & 74.6592 \\
\hline 18 & 999 & 35 & 2861 & 73.7936 & 89.3611 & 65.0836 & 82.8931 \\
\hline 18 & 999 & 45 & 2861 & 73.7049 & 74.0086 & 65.1433 & 67.9528 \\
\hline 18 & 999 & 40 & 2840 & 73.7488 & 80.5592 & 65.1145 & 74.2678 \\
\hline 18 & 999 & 40 & 2900 & 73.7497 & 81.6007 & 65.1139 & 75.3859 \\
\hline 26 & 1503 & 88 & 7526 & 69.3600 & 86.0126 & 63.4718 & 83.6187 \\
\hline 23 & 1503 & 88 & 7526 & 75.8186 & 86.0316 & 69.4822 & 83.5422 \\
\hline 29 & 1503 & 88 & 7526 & 63.9656 & 86.0114 & 58.4701 & 83.7024 \\
\hline 26 & 1450 & 88 & 7526 & 68.0226 & 86.0117 & 62.0660 & 83.6213 \\
\hline 26 & 1400 & 88 & 7526 & 66.7609 & 86.0109 & 60.7398 & 83.6240 \\
\hline 26 & 1525 & 88 & 7526 & 69.9152 & 86.0130 & 64.0554 & 83.6176 \\
\hline 26 & 1503 & 91 & 7526 & 69.3591 & 83.6080 & 63.4917 & 81.1899 \\
\hline 26 & 1503 & 85 & 7526 & 69.3611 & 88.5629 & 63.4513 & 86.1991 \\
\hline 26 & 1503 & 88 & 7520 & 69.3600 & 85.9574 & 63.4718 & 83.5609 \\
\hline 26 & 1503 & 88 & 7530 & 69.3600 & 86.0494 & 63.4718 & 83.6572 \\
\hline
\end{tabular}

reduction of number of failures observed, $k=9$ to $k=6$, in joint multiply type-II censoring scheme Bayes estimate of both the parameters decreases. The same concussions can be seen for reliability of the product also.

\section{Conclusion}

We have developed a maximum likelihood estimation of reliability for the products are being produced by two lines under the same facility based on multiply type-II joint censoring scheme. Bayesian estimation is considered using the importance sampling method. The effect of prior parameters on Bayes estimates is examined. The influence measure to identify observations which have disproportionately influence on MLEs of the parameters is also considered. 
Table 8 Bayes estimates of reliability at time $t=100$

\begin{tabular}{|c|c|c|c|c|c|c|c|}
\hline \multirow[t]{2}{*}{$c_{1}$} & \multirow[t]{2}{*}{$d_{1}$} & \multirow[t]{2}{*}{$c_{2}$} & \multirow[t]{2}{*}{$d_{2}$} & \multicolumn{2}{|l|}{$k=9$} & \multicolumn{2}{|l|}{$k=6$} \\
\hline & & & & $\hat{R}_{1}(t)$ & $\hat{R}_{2}(t)$ & $\hat{R}_{1}(t)$ & $\hat{R}_{2}(t)$ \\
\hline 18 & 999 & 40 & 2861 & 0.25770 & 0.29062 & 0.21529 & 0.26200 \\
\hline 15 & 999 & 40 & 2861 & 0.30158 & 0.29102 & 0.25847 & 0.26193 \\
\hline 21 & 999 & 40 & 2861 & 0.22067 & 0.29040 & 0.17988 & 0.26222 \\
\hline 18 & 1010 & 40 & 2861 & 0.25942 & 0.29062 & 0.21723 & 0.26200 \\
\hline 18 & 990 & 40 & 2861 & 0.25629 & 0.29062 & 0.21371 & 0.26200 \\
\hline 18 & 999 & 35 & 2861 & 0.25791 & 0.32659 & 0.21514 & 0.29928 \\
\hline 18 & 999 & 45 & 2861 & 0.25749 & 0.25893 & 0.21544 & 0.22956 \\
\hline 18 & 999 & 40 & 2840 & 0.25770 & 0.28900 & 0.21529 & 0.26015 \\
\hline 18 & 999 & 40 & 2900 & 0.25771 & 0.29362 & 0.21529 & 0.26540 \\
\hline 26 & 1503 & 88 & 7526 & 0.23651 & 0.31267 & 0.20690 & 0.30243 \\
\hline 23 & 1503 & 88 & 7526 & 0.26742 & 0.31275 & 0.23711 & 0.30210 \\
\hline 29 & 1503 & 88 & 7526 & 0.20944 & 0.31266 & 0.18082 & 0.30279 \\
\hline 26 & 1450 & 88 & 7526 & 0.22990 & 0.31266 & 0.19965 & 0.30244 \\
\hline 26 & 1400 & 88 & 7526 & 0.22360 & 0.31266 & 0.19275 & 0.30245 \\
\hline 26 & 1525 & 88 & 7526 & 0.23924 & 0.31267 & 0.20989 & 0.30243 \\
\hline 26 & 1503 & 91 & 7526 & 0.23651 & 0.30238 & 0.20701 & 0.29180 \\
\hline 26 & 1503 & 85 & 7526 & 0.23652 & 0.32331 & 0.20680 & 0.31345 \\
\hline 26 & 1503 & 88 & 7520 & 0.23651 & 0.31243 & 0.20690 & 0.30218 \\
\hline 26 & 1503 & 88 & 7530 & 0.23651 & 0.31282 & 0.20690 & 0.30260 \\
\hline
\end{tabular}

To exemplify the results obtained in the papers, two multiply type-II joint censored samples with different termination times $k=9$ and $k=6$ are considered from the real life example of failure times of air-conditioning systems in two airplanes 7914 and 7913.

We observe from the outcomes that maximum likelihood estimation of average failure time for plane 7914 is quite stable over different choice of $k$, and the estimation of average failure time for plane 7913 seems to be quite sensitive to the multiply type II censoring scheme employed when $k$ is small.

We have seen that for small sample i.e. $k=6$, no observations have siginifcant disproportionate infuenece on the MLEs.We also observe that increase in the value of shape parameter of prior distribution, Bayes estimate of mean failure time are sensitive and decreases in both the cases of censoring schemes, but remain almost stable under the changes in the value of scale parameter.

Abbreviations

MLE: Maximum likelihood estimate; ASE: Asymptotic standard error.

Acknowledgements

The author is thankful to the referees for their detailed comments and suggestions to improve the clarity of this paper. The author is also thankful to the editor of the journal.

Authors' contributions

I am a single author for this work and I have prepared it and approved the draft for publication. The author read and approved the final manuscript.

Funding

The author declares that she had no funding.

Availability of data and materials

The data set used for analysis is obtained from openly available in the journal. 


\section{Declarations}

Competing interests

The author declares that she has no competing interests.

Received: 29 August 2020 Accepted: 14 July 2021

Published online: 23 July 2021

\section{References}

1. Balakrishnan, N., Rasouli, A.: Exact likelihood inference for two exponential populations under joint type-Il censoring. Comput. Stat. Data Anal. 52(5), 2725-2738 (2008)

2. Balakrishnan, N., Feng, S.: Exact likelihood inference for $k$ exponential populations under joint type-II censoring. Commun. Stat.-Simul. Comput. 44, 591-613 (2014)

3. Shafay, A.R., Balakrishnan, N., Abdel-Aty, Y.: Bayesian inference based on a jointly type-Il censored sample from two exponential populations. Commun. Stat.-Simul. Comput. 43, 1-14 (2013)

4. Ashour, S.K., Abo-Kasem, O.E.: Bayesian and non-Bayesian estimation for two generalized exponential populations under joint type II censored scheme. Pak. J. Stat. Oper. Res. 10(1), 57-72 (2014)

5. Ashour, S.K., Abo-Kasem, O.E.: Parameter estimation for two Weibull populations under joint type II censored scheme. Int. J. Eng. Appl. Sci. 4(5), 31-36 (2014)

6. Parsi, S., Bairamov, I.: Expected values of the number of failures for two populations under joint type-II progressive censoring. Comput. Stat. Data Anal. 53, 3560-3570 (2009)

7. Rasouli, A., Balakrishnan, N.: Exact likelihood inference for two exponential populations under joint progressive typeII censoring. Commun. Stat.-Theory Methods 39(12), 2172-2191 (2010)

8. Balakrishnan, N., Su, F., Liu, K.Y.: Exact likelihood inference for k exponential populations under joint progressive typeII censoring. Commun. Stat.-Simul. Comput. 44(4), 902-923 (2015)

9. Nadi, A.A., Gildeh, B.S.: Estimating the lifetime performance index of products for two-parameter exponential distribution with the progressive first-failure censored sample. Int. J. Qual. Res. 10(2), 389-406 (2016)

10. Mondal, S., Kundu, D.: Inference on Weibull Parameters Under a Balanced Two Sample Type-Il Progressive Censoring Scheme. https://arxiv.org/abs/1801.00434, stat. AP. (2018)

11. Proschan, F.: Theoretical explanation of observed decreasing failure rate. Technometrics 5(3), 375-383 (1963)

12. Balakrishnan, N.: On the maximum likelihood estimation of the location and scale parameters of exponential distribution based on multiply type-II censored samples. J. Appl. Stat. 17, 55-61 (1990)

13. Balakrishnan, N., Gupta, S.S., Panchapakesan, S.: Estimation of the location and scale parameters of the extreme value distribution based on multiply type-Il censored samples. Commun. Stat. Theory Methods 24(8), 2105-2125 (1995)

14. Shah, J.B., Patel, M.N.: Bayes estimation under asymmetric loss functions multiply type II censored Rayleigh data. J. Indian Stat. Assoc. 46(1), 67-78 (2008)

15. Kim, C., Han, K.: Estimation of the scale parameter of the Rayleigh distribution with multiply type-II censored sample. J. Stat. Comput. Simul. 79(8), 965-976 (2009)

16. Upadhyay, S.K., Singh, U., Shastri, V.: Estimation of exponential parameters under multiply type II censoring. Commun. Stat.-Simul. Comput. 25(3), 801-815 (1996)

17. Kong, F.: Parameter estimation under multiply type-II censoring. Handb. Stat. 17, 315-335 (1998)

18. Shah, J.B., Patel, M.N.: Bayes estimation of a two-parameter geometric distribution under multiply type II censoring. Int. J. Qual. Stat. Reliab. (2011). https://doi.org/10.1155/2011/618347

19. Kang, S.B., Cho, Y.S., Han, J.T., Kim, J.: An estimation of the entropy for a double exponential distribution based on multiply type-II censored samples. Entropy 14,161-173 (2012)

20. Patel, D.T., Patel, M.N.: A Bayesian approach to optimal warranty length for exponential life time distributed product with multiply type-II censoring scheme. Int. J. Math. Stat. 18(3), 64-81 (2017)

21. Shafay, A., Sultan, K.: Bayesian inference based on multiply type-II censored samples of sequential order statistics from pareto distribution. J. Test. Eval. (2020). https://doi.org/10.1520/JTE20170699

22. Poon, W.Y., Tang, M.L.: Influence measure in maximum likelihood estimate for models of lifetime data. J. Appl. Stat. 28(6), 737-742 (2001)

23. Cook, R.D.: Assessment of local influence (with discussion). J. R. Stat. Soc. B. 48, 133-169 (1986)

24. Poon, W.Y., Poon, Y.S.: Conformal normal curvature and assessment of local influence. J. R. Stat. Soc. B. 61, 51-61 (1999)

25. Kundu, D., Pradhan, B.: Estimating the parameters of the generalized exponential distribution in presence of hybrid censoring. Commun. Stat. Theor. Methods 38, 2030-2041 (2009)

26. Rastogi, M.K., Tripathi, Y.M.: Inference on unknown parameters of a Burr distribution under hybrid censoring. Stat. Pap. 54, 619-643 (2013)

27. Mondal, S., Kundu, D.: A new two sample type-Il progressive censoring scheme. Commun. Stat. Theor. Methods 48(10), 2602-2618 (2019)

28. Sultana, F., Tripathi, Y.M., Rastogi, M.K., Wu, S.J.: Parameter estimation for the kumaraswamy distribution based on hybrid censoring. Am. J. Math. Manag. Sci. 37(3), 243-261 (2018)

\section{Publisher's Note}

Springer Nature remains neutral with regard to jurisdictional claims in published maps and institutional affiliations. 